\title{
Directional search for dark matter using nuclear emulsions with NEWSdm
}

\author{
Antonia Di Crescenzo*广 \\ University of Naples Federico II and INFN (IT) \\ E-mail: dicrescenzo@na.infn.it
}

\begin{abstract}
Cosmological observations indicate that most of the matter in the Universe is Dark Matter. Dark Matter in the form of Weakly Interacting Massive Particles (WIMPs) can be detected directly, via its elastic scattering off target nuclei. Most current direct detection experiments only measure the energy of the recoiling nuclei. However, directional detection experiments are sensitive to the direction of the nuclear recoil as well. NEWSdm is meant to be the first experiment with a solid target for directional dark matter searches: the use of a nuclear emulsion based detector, acting both as target and tracking device, would allow to extend dark matter searches beyond the neutrino floor and provide an unambiguous signature of the detection of Galactic dark matter.
\end{abstract}

An Alpine LHC Physics Summit (ALPS2018)

15-20 April, 2018

Obergurgl, Austria

\footnotetext{
* Speaker.

${ }^{\dagger}$ On behalf of the NEWSdm Collaboration
} 


\section{Introduction}

Several astrophysical and cosmological observations, involving different physical processes at diverse scales, provide evidence for the existence of non-baryonic Dark Matter in the Universe and, in particular, in the halo of our Galaxy, making up $27 \%$ of the total mass-energy density of the Universe [1]. These observations suggest that Dark Matter consists of at least one quasi-stable particle, that is not part of the Standard Model of particle physics. One of the best motivated particle Dark Matter candidates is a stable, massive, neutral particle generically referred to as a WIMP (Weakly Interacting Massive Particle) [2].

Several WIMP detection techniques have been proposed and are being pursued. Searches for laboratory-created WIMPs are ongoing at the Large Hadron Collider [3], as are attempts to detect Standard Model particles produced by WIMP annihilation in our own and extragalactic Dark Matter halos [4]. Direct detection experiments aim to detect Galactic halo WIMPs streaming through terrestrial detectors, via their elastic scattering off target nuclei.

As dark matter detectors are rapidly improving in sensitivity by approaching the ton-scale in mass, they will encounter the neutrino background, at which point Solar, atmospheric, and diffuse supernova neutrinos can mimic the dark matter signal. Neutrinos are therefore the ultimate background for WIMP direct detection searches as they cannot be shielded against and produce recoils with similar rates and energy spectra. Moreover, the recent controversy in the low-mass WIMP region $\mathrm{O}\left(10 \mathrm{GeV} / \mathrm{c}^{2}\right)$, where some dark matter hints are inconsistent with null results, highlights the need for additional discrimination power between WIMP events and backgrounds in order to clearly authenticate a genuine WIMP signal.

New generation detectors capable of measuring the direction of a nuclear recoil track resulting from the elastic scattering of a target nucleus by an incoming WIMP (Weakly Interacting Massive Particle), would provides a route towards the discrimination of neutrino background and the unambiguous identification of WIMPs as being responsible for the galactic dark matter.

From the experimental point of view several approaches have been proposed. The more diffuse strategy is based on the use of low pressure gaseous Time Projection Chambers (TPCs) [5]. Experiments based on this technology have provided the first directional limits in the Spin-Dependent (SD) case and the collaborations aim at developing larger detectors to be operated in a medium term time scale. Nevertheless this technology is hardly scalable to very large detectors masses needed to reach a good sensitivity to the Spin-Independent (SI) case.

The use of a solid target for directional searches would overcome the mass limitation of gaseous TPC approach thus allowing to reach an high sensitivity in the low cross section sectors of the SI case. Nevertheless, in a solid medium, the track of the WIMP-scattered nuclear recoil will have a path length of the order of a few hundred nanometers, much shorter than in the case of a gaseous target where the recoil length is expected to be of the order of a few millimeters. A detector with high tracking resolution is therefore needed.

\section{The NEWSdm experiment}

The approach proposed by the NEWSdm Collaboration [6] consists in the use of a nuclear emulsion-based detector acting both as target and as tracking device. The project foresees the 
employment of a novel emulsion technology called Nano Imaging Trackers (NIT) [7, 8] featuring a position resolution of an order of magnitude higher than that of the emulsion currently used in the OPERA experiment [9]. The detector is conceived as a bulk of NIT surrounded by a shield to reduce the external background. The detector is then placed on an equatorial telescope in order to absorb the Earth rotation, thus keeping fixed the detector orientation with respect to the incoming apparent WIMP flux. The angular distribution of the WIMP-scattered nuclei is therefore expected to be strongly anisotropic with a peak centered in the forward direction.

\subsection{NIT: Nano Imaging Trackers}

Nuclear emulsions are made of silver halide crystals embedded in a gelatine matrix. When ionizing particles pass through it, some of the halide crystals are modified in such a way that they are turned into grains of silver when a developing process is performed. The three-dimensional trajectory of passing through particles can be reconstructed with an optical microscope by connecting all the silver grains.

NIT are a novel type of emulsion films with grain diameters down to a few tens of nm, one order of magnitude smaller than conventional ones. They have a linear density of crystals of about 11 crystals $/ \mu \mathrm{m}[7]$ and they are made by the following nuclei (mass fraction in \%): $\mathrm{H}(1.6), \mathrm{C}(10.1)$, $\mathrm{N}$ (2.7), $\mathrm{O}$ (7.4), $\mathrm{S}(0.3), \mathrm{Br}(32.0), \mathrm{Ag}(44.0), \mathrm{I}(1.9)$. The total density is $3.2 \mathrm{~g} / \mathrm{cm}^{3}$. They make the reconstruction of trajectories with path lengths shorter than $100 \mathrm{~nm}$ possible, if analyzed by means of microscopes with enough resolution.

The presence in the emulsion gel of lighter nuclei such as carbon, oxygen and nitrogen, in addition to the heavier nuclei of silver and bromine, is a key feature of the NEWSdm project, resulting in a good sensitivity to WIMPs with both light and heavy masses.

\subsection{Read-out system}

In the NEWSdm experiment the expected WIMP signal will consist of short-path, anisotropically distributed, nuclear recoils over an isotropically distributed background. The search for signal candidates requires the scanning of the whole emulsion volume. The read-out system has therefore to fulfill two main requirements: a fast, completely automated, scanning system is needed to analyse the target volume over a time scale comparable with the exposure; the spatial resolution has to be improved by more than one order of magnitude compared to that achieved with standard emulsion films, reaching the challenging value of a few tens of nanometers, in order to ensure high efficiency and purity in the selection of signal candidates.

The analysis of NIT emulsions is performed with a two-step approach: a fast scanning with a state-of-the-art resolution for the signal preselection followed by a pin-point check of preselected candidates with unprecedented nanometric resolution to further enhance the signal to noise ratio and perform very accurate measurements of the range and the recoil direction. In the first phase a fast scanning is performed by means of an improved version of the optical microscope used for the scanning of the OPERA films $[11,13]$. In the last years an R\&D program aimed at improving the ESS performances was carried by INFN groups, leading to prototypes with resolution improved by one order of magnitude, achieving a speed of almost $200 \mathrm{~cm}^{2} / \mathrm{h}$ [12]. A new system is being developed in Japan (the Super-Ultra Track Selector), aiming at increasing the scanning speed up to $5000 \mathrm{~cm}^{2} / \mathrm{h}$. 
The starting point of the emulsion scanning is the image analysis to collect clusters made of dark grains at several depths across the emulsion plate thickness. Given the intrinsic resolution of the optical microscope $(\sim 200 \mathrm{~nm})$, the sequence of several grains making a track of a few hundred nanometers, appears as a single cluster. Therefore, the key element to distinguish clusters made of several grains from clusters made of a single grain produced by thermal excitation $(f o g)$ is the analysis of their shape. A cluster made of several grains indeed tends to have an elliptical shape with the major axis along the direction of the trajectory, while a cluster produced by a single grain tends to have a spherical shape.

In order to simulate the effect of a WIMP-induced nuclear recoil and to measure the efficiency and the resolution of the new optical prototype, a test beam with low velocity ions was performed. $\mathrm{Kr}$ ion beam with energies of 200 and $400 \mathrm{keV}$ [14] and a C ion beam with energies of 60, 80 and 100 $\mathrm{keV}$ were used.

When analysed with the optical microscope, submicrometric tracks produced by $\mathrm{Kr}$ and $\mathrm{C}$ ions appear as shown in Fig. 1(a). Although silver grains belonging to the tracks are not distinguishable and appear as a single cluster, the elongated form of the cluster is clearly visible [15]. An elliptical fit of the cluster shape allows a clear separation between fog grains and signal tracks: the latter ones are expected to have ellipticity larger than a given threshold, typically 1.25 or higher.

In order to evaluate the intrinsic angular resolution of the scanning system we analysed an emulsion sample exposed to a $2.8 \mathrm{MeV}$ neutron beam at the Fusion Neutronics Source (FNS) of the Japan Atomic Energy Agency (JAEA). An intrinsic angular resolution of $230 \mathrm{mrad}$ was achieved with fully automated scanning systems, by far the best resolution achieved with direction sensitive detectors in this energy range. The simulation shows that this result is compatible with the measurement reported above when the scattering contribution is included.

The second phase is performed exploiting the resonance effect occurring when nanometric metal grains are dispersed in a dielectric medium [16]. The polarization dependence of the resonance frequencies strongly reflects the shape anisotropy and can be used to infer the presence of nonspherical nanometric silver grains. NEWSdm will use this technology to retrieve track information in NIT emulsions beyond the optical resolution. Images of the same cluster taken with different polarization angles will show a displacement of the position of its barycenter. The analysis of the displacements allows to distinguish clusters made of a single grain from those made of two (or more) grains.

The evaluation of the position accuracy was performed by analysing images of single grains. The unprecedented accuracy of about $5 \mathrm{~nm}$ was achieved in both coordinates (Fig. 1(b)).

\section{State-of-the-art}

The NEWSdm collaboration is currently involved in an exposure test of $10 \mathrm{~g}$ detector. This exposure is meant to measure the detectable background from environmental and intrinsic sources and to validate estimates from simulations. The confirmation of a negligible background will pave the way for the construction of a pilot experiment with an exposure on the kg year scale or larger. The experimental setup is located at Gran Sasso underground laboratory and it consists of a shield from environmental backgrounds and a cooling system to ensure the required temperature level to the NIT emulsion detector. The optimization of the shield structure, along with the definition of the 


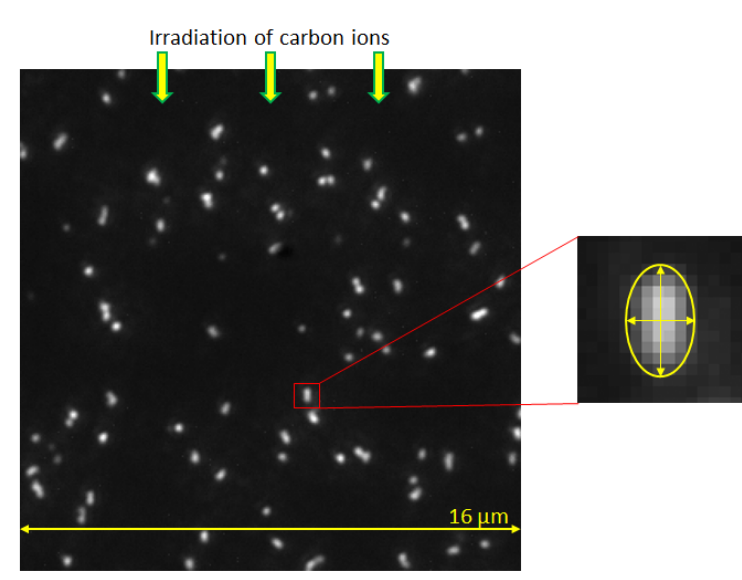

(a)

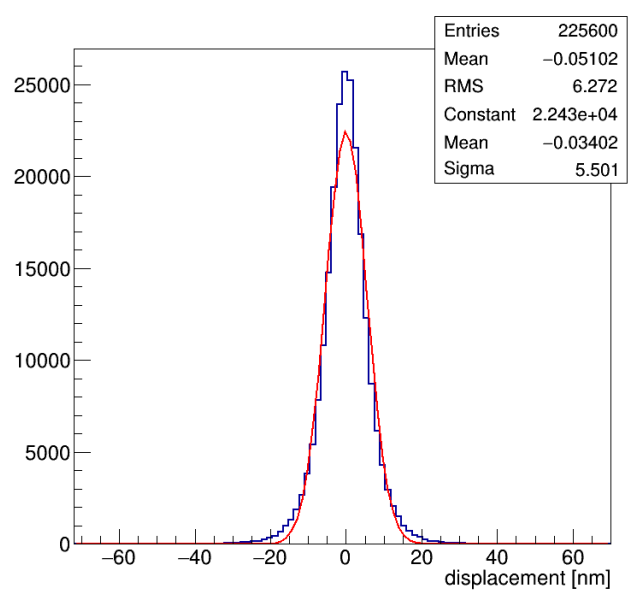

(b)

Figure 1: (a) Kr ions implanted on NIT films. The image is taken with an optical microscope. The selection of candidate tracks is based on the elliptic fit of the clusters. (b) Position accuracy of about $6 \mathrm{~nm}$ with the resonant light scattering.

material thickness, was performed with a Geant4 simulation. Results from simulation show that the combination of polyethylene and lead as shielding materials ensures a good rejection power from environmental neutrons and gammas, respectively. In particular, a thickness of $40 \mathrm{~cm}$ for the polyethylene blocks and of $10 \mathrm{~cm}$ for the lead plates was chosen to get a negligible contribution from environmental background. In Fig. 2 is reported a schematic representation of the shield (left) and the whole structure installed in Hall B (right).
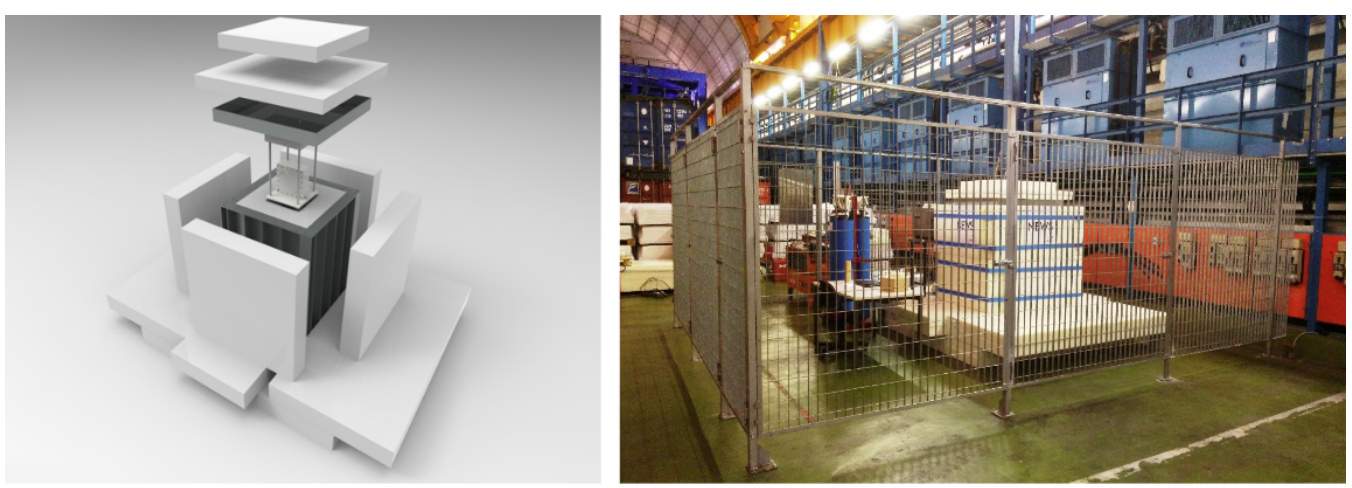

Figure 2: Shield explxoded view (left) and a picture of the detector installed in Hall B (right).

\section{Sensitivity using directionality}

The discovery potential of an nanometric-emulsion-based detector to distinguish a WIMP signal from the background, profiting of the signal anisotropy over an isotropic background has been recently studied in the SHM case [19]. The capability of measuring track lengths above $100 \mathrm{~nm}$ 


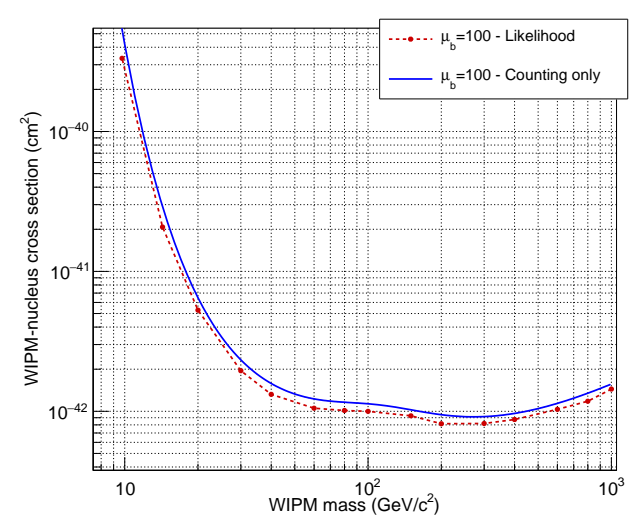

(a)

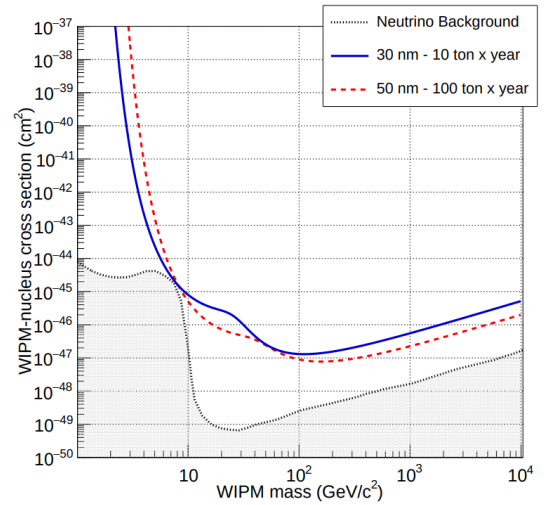

(b)

Figure 3: (a) Exclusion curve at 90\% confidence for WIMP in the mass-crosssection plane for 100 expected background events and $100 \mathrm{~kg}$ year exposure. (b) Exclusion limits for the NEWSdm detector with 10 ton $\times$ year exposure and $30 \mathrm{~nm}$ threshold (solid blue curve) and with 100 ton $\times$ year exposure and $50 \mathrm{~nm}$ threshold (dashed red curve).

without sense recognition was assumed. The angular distribution of WIMP-induced recoils is expected to have a Gaussian shape peaked at zero, with a sigma depending on the WIMP mass. The lighter the WIMP, the stronger the angular anisotropy. Indeed, for low WIMP masses the recoil energy is rather low and the track length threshold selects only a small fraction of the spectrum, characterised by the largest fractional energy transfer to the recoiled nucleus. This makes the sigma of the angular distribution narrower, thus enhancing the directional feature. The estimate of the expected significance for a Dark Matter search with a directional detection in emulsions was performed following a frequentest approach with the profile likelihood ratio test [20]. The power of the directionality was evaluated in absence of a signal assuming $100 \mathrm{~kg}$ year exposure and 100 background events. The upper limit evaluation was performed fixing the CL at $90 \%$ and applying the CLs correction. The curve is obtained by plotting the exclusion limits for different WIMP masses and it is reported in Fig. 3(a) using both the Likelihood Ratio (red) and Poisson methods (blue). The gain obtained exploiting the directional information with respect to the Poisson method ranges from $10 \%$ to $20 \%$. In Fig. 3(b) the NEWSdm exclusion limit assuming zero background is compared with the curve representing the neutrino bound for a Xe/Ge target (gray dotted curve), as evaluated in [21]. The neutrino limit is reached with a 10 (100) ton $\times$ year exposure if a 30 (50) $\mathrm{nm}$ threshold is achieved. The resonance effect of polarised light even now allows to reach an accuracy in the position of the grains less then $10 \mathrm{~nm}$ in both $\mathrm{X}$ and $\mathrm{Y}$ coordinates (see Sec. 2.2). Besides, nano-metric grains of $\sim 20 \mathrm{~nm}$ of size are already developed in Nagoya University paving the way for a reduction of the detector threshold. On the other side, the scanning speed performances of fully automated optical microscopes is rapidly increasing in the last years allowing in the near future the design of a ton-scale detector-based on NIT emulsions. 


\section{Conclusions}

The novel emulsion technology, based on the use of nuclear emulsion with nanometric $\mathrm{AgBr}$ crystals (NIT), makes it possible to record the sub-micrometric tracks produced by the WIMP scattering off a target nucleus. The presence, in the emulsion components, of light and heavy nuclei results in an enhanced sensitivity to both light and heavy WIMP masses. The final signal confirmation is obtained with powerful optical microscope equipped with a light polarizer: exploiting the different response of non spherical grain clusters to different polarization angles, the unprecedented spatial resolution of $\sim 6 \mathrm{~nm}$ is obtained. A $10 \mathrm{~g}$ exposure test is in progress at Gran Sasso underground laboratories.

\section{References}

[1] P.A.R. Ade et al., arXiv:1502.01589

[2] G. Bertone, D. Hooper and J. Silk, Phys. Rept. 405 (2005) 279

[3] E. Halkiadakis, G. Redlinger and D. Shih, Ann. Rev. Nucl. Part. Sci. 64 (2014) 319

[4] T. A. Porter, R. P. Johnson and P.vW. Graham, Ann. Rev. Astron. Astrophys. 49 (2011) 155

[5] J.B.R. Battat et al., Phys. Rep 662 (2016) 1

[6] A. Alexandrov et al. (NEWS Collaboration), arXiv:1604.04199, LNGS-LOI 48/15

[7] M. Natsume et al., Nucl. Instr. Meth. A575 (2007) 439

[8] T. Naka et al., Nucl. Instrum. Meth. A718 (2013) 519

[9] A. Agafonova et al. (OPERA Collaboration), JINST 4 (2009) P04018.

[10] B. Morgan, A.M. Green and N.J.C. Spooner, Phys. Rev. D 71 (2005) 103507

[11] N. Armenise et al., Nucl. Instr. Meth. A551 (2005) 261

L. Arrabito et al., Nucl. Instr. Meth. A568 (2006) 578

[12] A. Alexandrov, V. Tioukov, M. Vladymyrov, JINST 9 (2014) C02034

[13] K. Morishima and T. Nakano, JINST 5 (2010) P04011

S. Aoki et al., Nucl. Instrum. Meth. B 51 (1990) 466

[14] T. Naka et al., EAS Publ. Ser. 53 (2012) 51

[15] M. Kimura and T. Naka, Nucl. Instrum. Meth. A680 (2012) 12

[16] H. Tamaru et al., Applied Phys. Lett. 80 (2002) 1826

[17] W.B. Wilson et al., LA-13639-MS, Los Alamos (1999)

[18] A. Alexandrov et al., Astropart. Phys. 80 (2016) 16

[19] A. Agafonova et al. (NEWSdm Collaboration), Eur. Phys. J. C 78 (2018) no.7, 578

[20] G. Cowan, K. Cranmer, E. Gross and O. Vitells, Eur. Phys J. C71 (2011) 1554, [Erratum: Eur. Phys. J. C73 (2013) 2501]

[21] J. Billard, E. Figueroa-Feliciano and L. Strigari, Phys. Rev. D 89 (2014) 023524 